\title{
LOCAL PARAMETERS OF AIR-WATER TWO-PHASE FLOW AT A VERTICAL T-JUNCTION
}

\author{
G. Monrós-Andreu, R. Martínez-Cuenca, S. Torró and S. Chiva \\ Universitat Jaume I \\ gmonros@uji.es; rcuenca@uji.es; torro@uji.es; schiva@uji.es
}

\begin{abstract}
Significant experimental work and modeling about vertical T-junction as a phase separator has been done for churn and annular flows, but a survey on the literature reveals a lack of experimental data regarding bubbly flow nor any phenomenological explanation to their behavior. The objective of this work is to extend the understanding of these junctions by obtaining complete datasets, i.e. of both gas and liquid, of the phase splitting process in bubbly flow conditions by means of conductivity needle probes, Laser Doppler anemometry and visual inspection. Measurements and observations of the phase split, as well as the vortex structure in a vertical T-junction with equal pipe diameters (52 $\mathrm{mm}$ inner diameter), are reported. Results suggest a relationship between the vortex structure and the efficiency of the junction as phase separator.
\end{abstract}

KEYWORDS

Two-phase, T-junction, vertical pipe, conductivity probes, LDA

\section{INTRODUCTION}

Two-phase gas-liquid flow in T-junctions takes place in numerous industrial systems and process plants as in oil extraction industry or water cooled nuclear reactors. Consequently, the study of their behavior and a proper modeling of their dynamics is of great interest. This modeling can be performed through onedimensional codes (RELAP5, etc) that are based on the use of empirical correlations after the study of an extensive set of experimental conditions. But in order to understand and optimize their performance the more detailed three-dimensional Computational Fluid Dynamics (CFD) codes are needed. These studies require the development of new databases with more information on the local distribution and properties of liquid and gas within the junction for a proper validation of the two-phase flow models.

In short, T-junctions can be used in two ways when applied to two-phase flow systems. First, they can act simply as flow diverters, so the flow characteristics are expected to be similar in both branches after the junction. Second, they can be used as phase separators (Azzopardi et al., 2002) so that one of the phases must be completely removed from one of the branches. In order to accomplish with the two tasks, it is essential to understand and be able to predict the degree of phase segregation and general flow behavior of the mixture at T-junctions for various operating conditions. The present state of understanding reveals the great complexity of analyzing the segregation phenomena, with the most important parameters being the inlet flow pattern (Azzopardi, 1982), radial void fraction distribution (Azzopardi, 1984) and branchinlet diameter ratio (Charron and Whalley, 1995). Of course, phase-density difference is critical (Issa and Oliveira, 1994). In case of noticeable density differences between phases, as in air-water mixtures, the lighter phase tends to be diverted into the side arm whereas the heavier one tends to flow in the inlet

Corresponding author 
branch direction. As the densities become similar, this effect is clearly reduced towards the limit of equalphase density, where the flows tend to distribute quite uniformly.

This paper presents a study on the local two-phase distribution and mechanics in a T-junction for four experimental conditions. Two different inlet superficial liquid velocities ( 2 and $3.3 \mathrm{~m} / \mathrm{s})$ for two different gas superficial velocities. Selected conditions lead to different void distributions (center-peaked and wallpeaked profiles) at T-junction inlet, thus providing a variety of scenarios useful to study the mixing behavior. Detailed information regarding discrete phase is followed by a preliminary study of the continuous phase at splitting region inside the T-junction. Both liquid (velocity and turbulence intensity) and gas (void fraction, interfacial velocity, interfacial area concentration and bubble frequency) data are provided. Next section will provide a description of the experimental facility used to measure the flow characteristics through a T-junction. Section 3 aims at briefly describing the four-sensor conductivity probes used to measure the dispersed-gas phase characteristics. The experimental results for the flow conditions investigated are presented in Section 4. Besides of conductivity probe results, Section 4 include the continuous phase measurements performed by using Laser Doppler Anemometry (LDA) technology. We refer to (Zhang, 2010) for more detailed and general information on the use of this technique. After showing the feasibility of the proposed methodology in sections 3 and 4, we summarize the main results in the Conclusions.

\section{EXPERIMENTAL FACILITY}

As illustrated in Figure 1, the experimental facility consists of two vertical and one horizontal transparent Plexiglas ${ }^{\circledR}$ pipe sections with $52 \mathrm{~mm}$ inner diameter (ID). The three sections are connected with a Tjunction built from two symmetrical mechanized parts, one of them made of Plexiglas ${ }^{\circledR}$ to enable optical access into the flow.
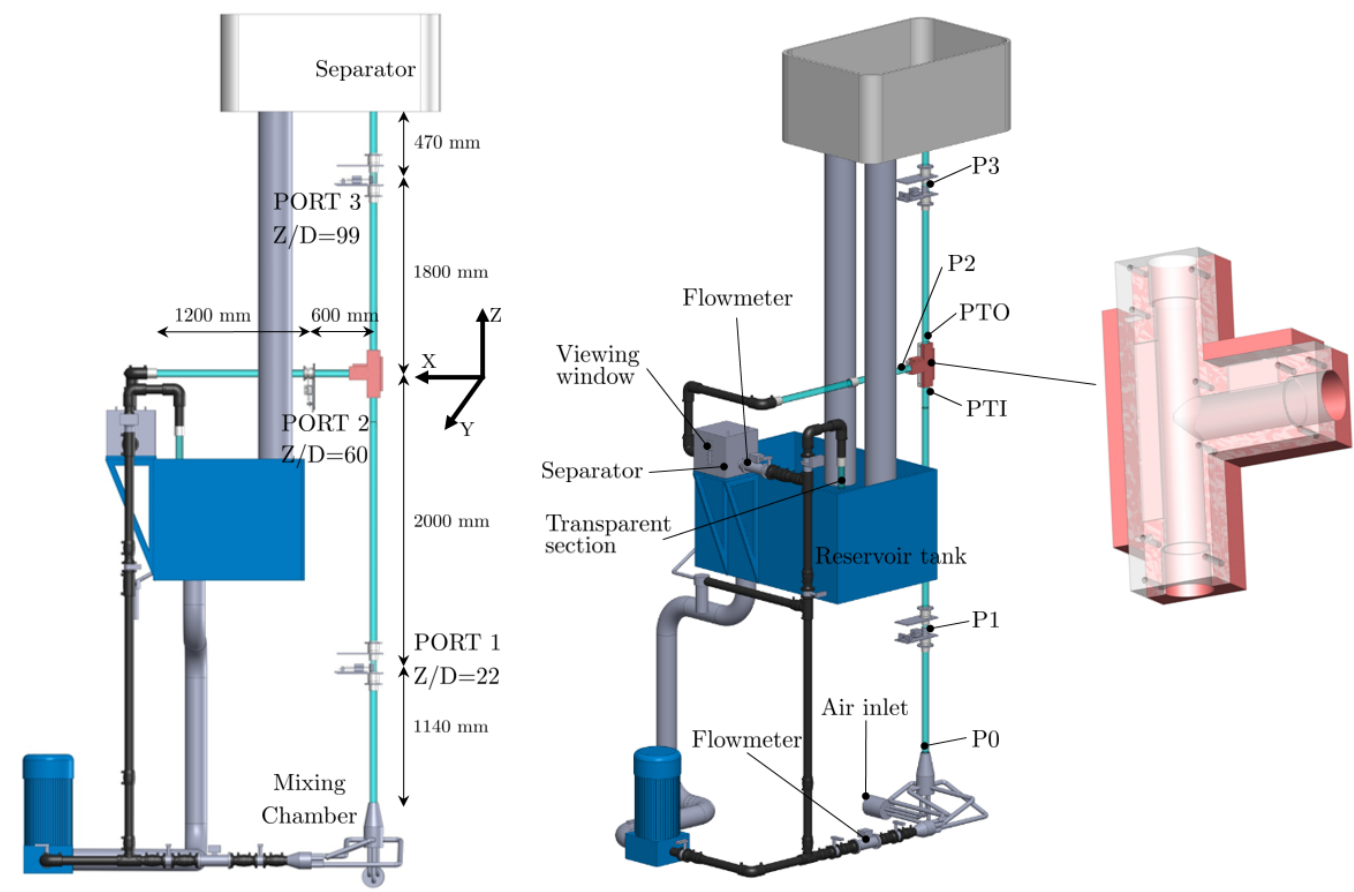

Figure 1. Schematic diagram of the experimental facility and a detail of the T-junction.

Operating fluids are dried and filtered air and purified water obtained from an osmotization process (conductivity $80 \mu \mathrm{S} / \mathrm{m}$, controlled to be constant and to detect any possible contamination during the 
experiments). The water is circulated by a centrifugal pump and stored in a 500L reservoir tank and is kept at a constant temperature $\left(20^{\circ} \mathrm{C}\right)$ thanks to a heat exchanger. An air flow-meter controller (Bronckhorst EL-FLOW $250 \mathrm{lNpm}$ ) is used to adjust and measure the gas flow-rate.

The air-water mixing is performed in a mixing chamber at the bottom of the facility. This chamber is composed by 4 separate compartments, each one having a liquid inlet at the bottom and a gas inlet through a sparger $(10 \mu \mathrm{m}$ average porosity) that is oriented axially with the main flow. This configuration provides a quite uniform distribution of gas and liquid at the pipe inlet. After passing through the Tjunction, the flow is diverted in two branches, each one having a phase separator at its end. The one located on the top of the vertical section is a deposit opened to atmosphere. The gas just comes out to the air and the liquid falls down to the main reservoir tank. The separator after the horizontal branch has interior baffles to improve the separation and a viewing window to control the water level. The air from the separator tank passes through two separator valves and a small air-reservoir tank to smooth air pressure fluctuations.

Both liquid and gas flow rates were measured at the bottom of the facility (before entering the mixing chamber) and after the separator in the horizontal branch. Also, water temperature was measured in the reservoir tank and before the mixing chamber by using PT100 thermocouples. Pressure was measured with pressure transducers with a range $0-1$ bar for P0 and P1 locations, 0-0.6 Bar for PTI, PTO and P2 locations and $0-0.25$ bar for $\mathrm{P} 3$ location (see Figure 1 for these locations).

The local flow parameters were measured at three measurement ports. Port 1 was located in the first vertical section, close to the inlet $(Z / D=22)$. Port 2 was located at the horizontal branch $(Z / D=60, X / D=10$ from the center of T-junction). Port 3 was located at the second vertical section $(Z / D=99)$.

Gas-phase local parameters were measured using four-sensor conductivity probes, mounted on each measurement port. As shown in Figure .a, the conductivity probes are attached to a scanning system driven by a stepper motor. This system is used to perform the gas phase measurements at 15 radial locations along the section radius $(0,5,8,11,16,20,21,21.5,22.5,23,23.5,24,24.5,25$ and 25.5 millimeters) as depicted in Figure 2.b and Figure 2.c. As local flow parameter variations are expected to be higher close to pipe wall, small increments for radial measurement locations are used in this zone to improve spatial resolution. Azimuthal symmetry of flow distribution was assumed as the measurement ports are far from the T-junction. Port 2 was constructed on a rotating section to enable measurements across the whole pipe section as phase stratification breaks azimuthal symmetry for this case (symmetry about the XZ plane was assumed instead). Both axisymmetric assumption for the bubble distributions (for horizontal and vertical sections) were previously confirmed via measurements of local void fraction and bubble frequency. As such, the local measurements are obtained only along one azimuthal angle for vertical port and one-half of the section for horizontal port. The rotating section (Figure 2.a, right side) can be manually rotated in $30^{\circ}$ steps intervals in the azimuthal direction during the experiment without stopping the flow. The three measurement ports provide a total of 135 distinct measurement locations for each experiment (see Figure 2.c). More details on the measurement procedure with these probes will be addressed in Section 3.

a)

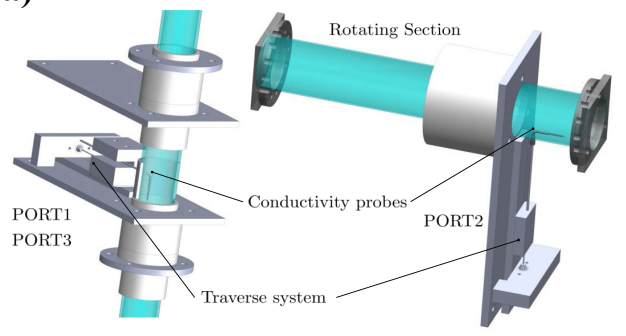

b)

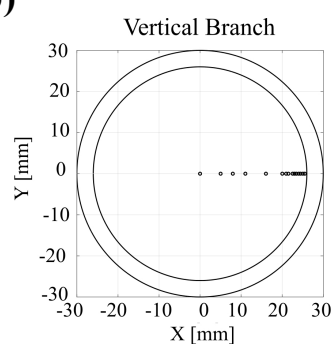

c)

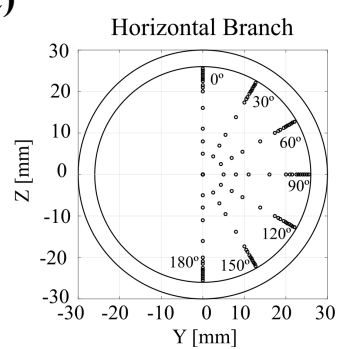


Figure 2. Detail of measurement ports and measurement points.

In order to measure the liquid phase velocity and turbulence intensity inside the T-junction, a backscattering LDA probe is used. The LDA equipment was composed of a $0.5 \mathrm{~W} \mathrm{Ar}^{+}$Ominichrome Laser, a beam separator (Dantec Fiberflow), a signal processor (Dantec FVA 58N40) and acquisition software (Floware). Two laser lines were used (488 and $512 \mathrm{~nm}$ with a focal spot of approximately $0.6 \mathrm{~mm}$ width and $2 \mathrm{~mm}$ depth). Round polyamide seeding particles (mean diameter of $5 \mu \mathrm{m}$ ) were dispersed in the water in such a concentration that the sampling frequency remained over $1 \mathrm{kHz}$ for single-phase conditions.

To reach quasi-stationary flow and precise measurements of air-separated flow rate, the pressure inside the separator tank is controlled manually with the rotameter aperture until the water level remained constant for about 30 minutes before each run test. Probe voltage signals were simultaneously recorded over 15 seconds for all experimental conditions at a $60 \mathrm{kHz}$ individual tip sampling rate $(720 \mathrm{KHz}$ total sampling rate considering the three sensors) by using a high-speed acquisition board (NI SCXI-1325). Probe positioning, flow rates setup and variable measurement acquisition are implemented and automated in LabView ${ }^{\circledR}$ environment.

\section{FOUR-SENSOR PROBE METHODOLOGY}

The existing literature for needle probe sensors (Kataoka et al., 1986; Kataoka and Serizawa, 1990; Revankar and Ishii, 1992; Kalkach-Navarro et al., 1993; Cartellier, 1999; Dias et al., 2000; Fu, 2001; Wu et al., 2001; Kim et al., 2001; Euh et al., 2001; Shen et al., 2005; Pradhan et al., 2013) is employed to measure the local two-phase flow parameters in the present work. The four-sensor conductivity probe design used in the present experiments is based in the previous work of (Kim et al., 2001) and consisted of 4 enamelled copper wires $(0.2 \mathrm{~mm}$ outer diameter). The wires were glued together by means of an epoxy resin so that the axial distance between front and rear wire tips was set around $2 \mathrm{~mm}$ and maximum radial distance between them about $0.5 \mathrm{~mm}$. Figure 3.b depicts the tip arrangement and typical probe dimensions used. Only the very end of each wire is electrically exposed to surrounding media.

The four wires were hold by a metallic stainless steel tube kept at ground potential. The probe holder and each of four sensor tips act as a negative and positive electrode respectively of a close circuit and provide the measuring voltage. Basically each wire works independently as a phase identifier, giving information about the phase surrounding each tip: presence of conductive media (water) is interpreted as a close circuit and the presence of non-conductive media (air) as an open circuit. This fact is used to provide four independent voltage signals related to the phase present.

As depicted in Figure 3.a, the front tip (larger wire tip, labeled as "0") serves as origin of coordinates for the tip locations of the three rear tips (labeled as "1", "2" and " 3 "). A set of distance vectors between front and rear tips $\left(\overrightarrow{s_{01}}, \overrightarrow{s_{02}}, \overrightarrow{s_{03}}\right)$ is then obtained. According to (Kataoka et al., 1986), calculation of two-phase flow parameters only requires that geometry vectors (sensor tip coordinates) and the time delays between the four voltage signals from detected bubble interfaces. 
a)

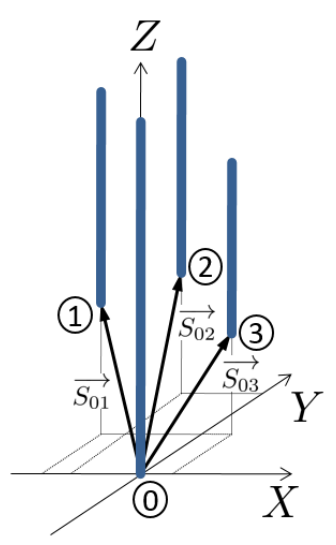

b)

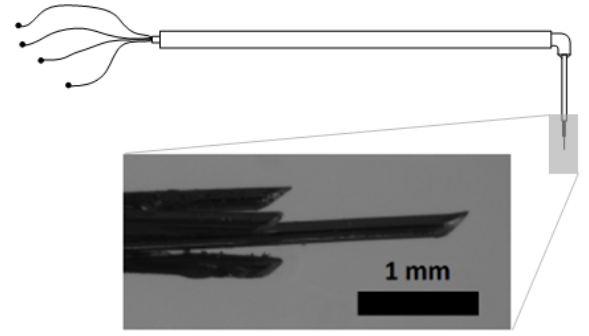

$\vec{S}_{01}=\left[\begin{array}{lll}0.04 & 0.43 & 1.92\end{array}\right] \mathrm{mm}$

$\vec{S}_{02}=\left[\begin{array}{llll}0.29 & 0.02 & 1.91\end{array}\right] \mathrm{mm}$

$\vec{S}_{03}=\left[\begin{array}{lll}0.28 & 0.27 & 1.85\end{array}\right] \mathrm{mm}$

Figure 3. Probe tip arrangement (a), typical conductivity dimensions probe and detail of the sensing tips $(\mathbf{b})$.

However, raw signal outputs from the sensing probes are far from being ideal two-state square-waves. This is caused by the finite size of the sensors and the time delay needed to wet or rewet the sensing tips. In order to obtain a square-wave signal representing the phase indicator function, a threshold method proposed by (Barrau et al., 1999) combined by method proposed by (Dias et al., 2000) was used in the present work. Figure 4 shows an example of such raw signals and the reconstructed phase-indicator functions corresponding to the four sensing tips. A set of delay times are then obtained representing the time arrivals and departures of the individual bubbles, and thus, the gas phase duration. Local gas parameters can be computed basing on these time delays and sensor tip coordinates.

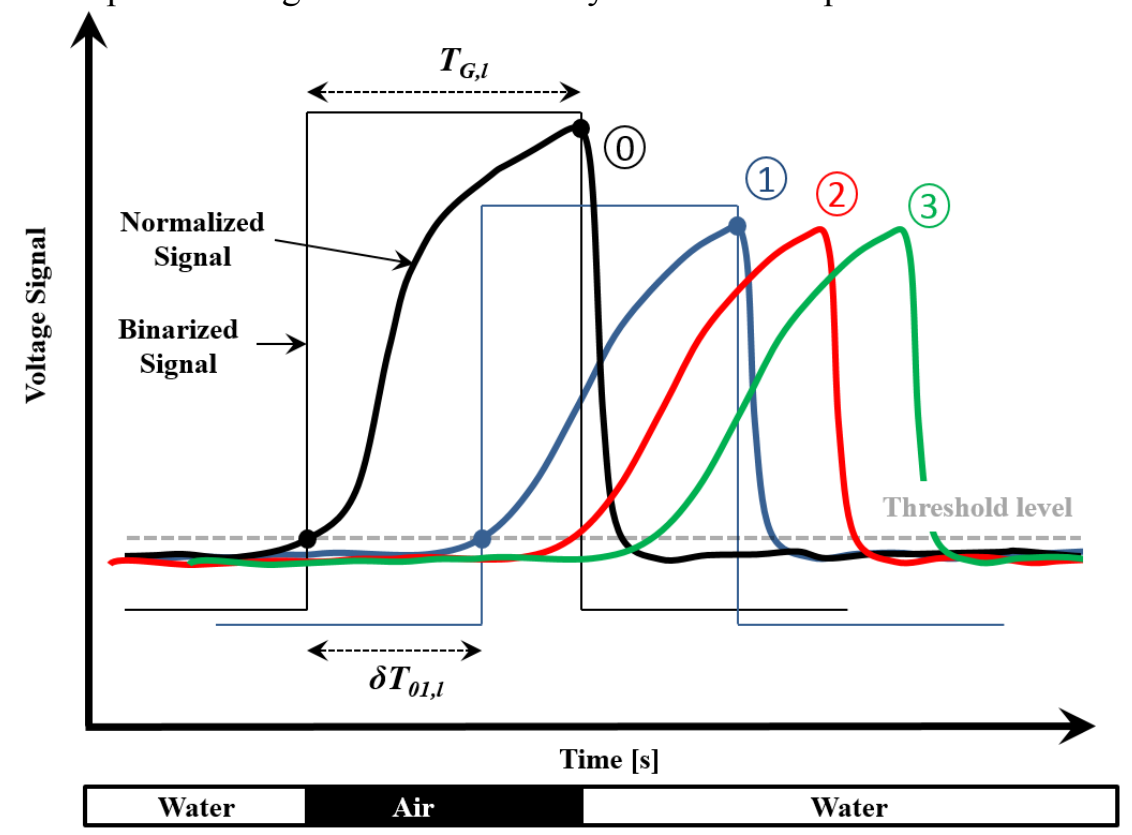

Figure 4. Example of main objective parameters to be obtained from signals.

In what follows, the procedure to calculate the main parameters needed to characterize the gas phase, such as the void fraction, the velocity of the bubble's interfaces, the bubble frequency, bubble size (estimated by chord length) and the interfacial area concentration, is presented: 
a) Void fraction:

The mean local volume fraction of the bubbles (or time-averaged void fraction, $\alpha$ ) at the position of the probe can be well estimated from the conductance signal, as it can be determined by the fraction of time that the sensor is exposed to gas phase $\left(T_{G, L}\right)$ over the total sampling time $(\Omega)$.

$$
\alpha=\frac{\sum T_{G, l}}{\Omega}
$$

Void fraction is computed by using the signal corresponding to front sensor as it causes less disturbances in the bubble interface piercing process.

b) Interfacial velocity:

The time delay for the paired signals between two needles, say front tip (0) and a rear one $(k=1,2$, or 3$)$, allows for the calculation of the interfacial velocities of individual bubbles. If each bubble is labeled with the sub-index $l$, then the $l$-th corresponding bubble interfacial velocity parallel to the flow direction, $\overrightarrow{V_{0 k, l}}$ can be computed from corresponding distance vector, $\overrightarrow{s_{0 k, l}}$ (Tian et al., 2014) as:

$$
\overrightarrow{V_{0 k, l}}=\frac{\overrightarrow{s_{0 k, l}}}{\delta T_{0 k, l}}, k=1,2,3
$$

Where $\delta T_{0 K}$ stands for the time delay between the rising time of central tip and the rising time of rear tip $k$ (Figure 4 ). In this work we have computed interfacial velocity parallel to the flow direction as the average of individual velocities obtained from previous equation (Xue, 2004):

$$
\overrightarrow{V_{l, l}}=\frac{\left|\overrightarrow{V_{01, l}}\right|+\left|\overrightarrow{V_{02, l}}\right|+\left|\overrightarrow{V_{03, l}}\right|}{3}
$$

Even in a vertical upward flow configuration the velocity of air bubbles are not purely axial but have small lateral components as well. (Wu et al., 2001) demonstrated that bubbles with significant lateral motion can cause the surface of a bubble to strike almost simultaneously the front and rear sensor tips (like piercing bubble near to the edge), so it produces a very small time delay and thus an overestimation of axial velocity component. This bubble lateral motion can also increase the number of missing bubble (bubbles which touch the front sensor tip but miss one or more rear sensor tips). Missing bubbles are not taken into account for interfacial velocity. Missing bubbles issues implies that the right selection of two closely corresponding signals from each sensor is mandatory, because sequential signals detected by the four sensing tips do not always correspond to the same bubble interface. In order to overcome those problems, in this work it is suggested the use of two velocity thresholds to validate the detected bubbles, which is a similar methodology proposed by (Revankar and Ishii, 1992) and (Guet et al., 2003).

The two velocity thresholds (upper and lower) are based on the mean cross-correlation velocity $\left(v_{c o r r}\right)$ used as a reference velocity, obtained by using the axial distance between front and rear tips and the most probable transport time between signals. Three most probable transport times are obtained, as in (Liu and Bankoff, 1993), by cross-correlation from front sensor signal and each $k$ rear sensor (and thus the associated most probable 
velocity, $\left.v_{c o r r}, 0 k\right)$ signal. We used the whole acquired and normalized signals to perform the cross-correlation. Thereafter, $v_{\text {corr }}$ is computed as:

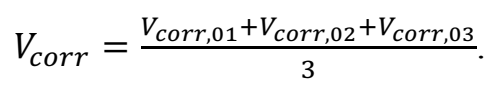

A $C_{f}$ factor set to 3 is used for current experiments to set the thresholds in Eq. 5 (admissible bubble velocities three times larger or smaller than $v_{c o r r}$ ). Threshold limits proposed in are wide enough just to prevent extreme values affecting averaged velocity (under 14\% of total computed bubbles for tested conditions).

$$
\left(1 / C_{f}\right) v_{c o r r}<\overrightarrow{V_{l, l}}<C_{f} v_{c o r r}
$$

The mean value of interfacial velocity is obtained from the log-normal PDF fitting of filtered $\overrightarrow{V_{l, l}}$ data which satisfy Eq. 5 .

c) Interfacial area concentration:

The IAC is defined by the total interfacial area per unit mixture volume. Up to now, several investigations and experimental measurements have been carried out using four sensor probe methodology, proving the consistency of this method for IAC measurements. From the pioneer work of (Kataoka et al., 1986) on the local interfacial area derivation from velocity measurements, (Kim et al., 2001) proposed a miniaturized probe and made it capable of measuring both large and small bubbles. (Shen et al., 2005) and (Shen and Nakamura, 2013) proposed an explicit equation for the instantaneous local interfacial area and local interfacial velocity using four sensor conductivity probes, which take into account both receding and oncoming interfaces. Then, local values of IAC can be calculated as:

$$
\bar{a}_{i}^{t}=\frac{1}{\Omega} \cdot \sum_{l=1}^{2 N_{t}} \sqrt{\frac{D_{01 l^{2}+D_{02 l}^{2}+D_{03 l} l^{2}}}{D_{0}^{2}}}
$$

Where $N_{t}$ denotes the measured bubble number within the total sampling time $\Omega$, and $D_{0}, D_{01 l}$, $D_{02 l}$ and $D_{03 l}$ are the basic and directional determinants related to the four-sensor probe, totally determined by the three rear sensor tip coordinates referenced to front sensor tip coordinates and the time delays between square-wave signals (Figure 4). These directional determinants are expressed by:

$$
\begin{aligned}
& D_{0}=\left|\begin{array}{lll}
x_{1} & y_{1} & z_{1} \\
x_{2} & y_{2} & z_{2} \\
x_{3} & y_{3} & z_{3}
\end{array}\right| \\
& D_{01 l}=\left|\begin{array}{lll}
\Delta t_{01 l} & y_{1} & z_{1} \\
\Delta t_{02 l} & y_{2} & z_{2} \\
\Delta t_{03 l} & y_{3} & z_{3}
\end{array}\right| ; D_{02 l}=\left|\begin{array}{lll}
x_{1} & \Delta t_{01 l} & z_{1} \\
x_{2} & \Delta t_{02 l} & z_{2} \\
x_{3} & \Delta t_{02 l} & z_{3}
\end{array}\right| ; D_{03 l}=\left|\begin{array}{lll}
x_{1} & y_{1} & \Delta t_{01 l} \\
x_{2} & y_{2} & \Delta t_{02 l} \\
x_{3} & y_{3} & \Delta t_{03 l}
\end{array}\right|
\end{aligned}
$$

As interfacial velocity, time-local averaged IAC considers only effective interfaces (detected by the four sensor tips) and can be computed with Eq. (6). For missing bubbles/interfaces it is not possible to measure their velocity and therefore their contribution to IAC, and there is no accurate theoretical way to evaluate them in the measurement. In the classical procedure to overcome this problem in bubbly flow, the missing bubbles/interfaces are treated as if they possess the average value of IAC. (Wu and Ishii, 1999) and (Le Corre and Ishii, 2002) found 
numerically that this approach underestimates the real value of expected IAC, considering the fact that missing bubbles are generally the smallest bubbles or they are caught in the edge, and these ones provides the major contribution to IAC. In this work, the results for the time-local averaged IAC were corrected by the method proposed by (Le Corre and Ishii, 2002):

$$
a_{i, \mathrm{CORR}}=\frac{1}{\sqrt{1-\sqrt{2.4 R_{N}-1.5 R_{N}^{2}}}} \cdot a_{i, M E A S} \quad R_{N} \in[0-0.7]
$$

Where $a_{i, C O R R}$ and $a_{i, M E A S}$ are the corrected time-local averaged IAC and the time-local averaged IAC from computed bubbles/interfaces. The correction method only takes into account the bubble missing ratio, $R_{N}$, as the ratio between the number of bubbles classified as missing and the total number of detected bubbles (computed with front sensor).

Next figure shows the missing ratio (mean and standard deviation) for all local measurements (1260 positions). As expected, missing ratios toward the center of the pipe section are lower for all cases. Except at the two closest radial positions to pipe wall, where the probability of piercing bubbles near to its edge is higher, good efficiency in bubble detection rates are achieved for probe dimensions used. Moreover, it confirms the applicability of Eq. 8 for IAC correction in the presented run tests.

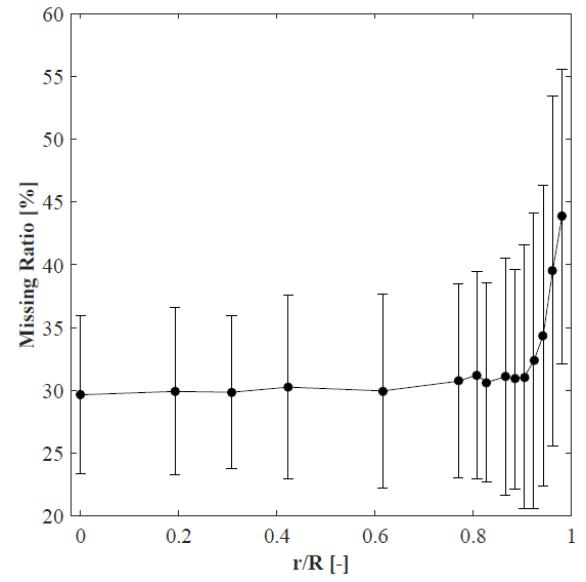

Figure 5. Local missing ratios (mean and deviation) obtained for all experiments performed.

d) Chord length

Here the bubble chord length is defined as the product of the bubble velocity and the bubble transit time. Bubble individual velocity is obtained according to Eq. (3), and the corresponding transit time is obtained from the pulse duration of the front sensor, which provides the less affected signal by bubble probe interaction. Hence the bubble chord length is related to bubble size. Mean chord length values are obtained from the log-normal fit of the obtained PDF.

e) Bubble frequency

The bubble frequency at each local point is computed as the number of bubbles detected per unit time by the front sensor of four-sensor conductivity probe (number of rising edges in the corresponding binarized signal). This parameter could provide, in conjunction with local void fraction, qualitative information about bubble coalescence and breakage phenomena. 


\section{EXPERIMENTAL RESULTS: LOCAL FLOW PARAMETERS}

In this study, four two-phase flow conditions have been tested corresponding to two liquid flow rates, and two gas conditions for each liquid flow rate. The parameters defining these conditions are summarized in Table I. Superficial velocities for liquid phase $\left(j_{L}\right)$ at $\mathrm{z} / \mathrm{D}=0$ (inlet) and horizontal branch are listed. Local superficial gas velocity $\left(j_{G}\right)$ is calculated based on gas volumetric flow rate measurement and the pressure at $\mathrm{Z} / \mathrm{D}=22$ (Port1). Area-averaged values are denoted by $<>$. Table I also includes the area-averaged void fraction measured with the four-sensor conductivity probe, although it is not a setup variable, is important variable to define the experimental condition.

Table I. Experimental test conditions (Setup variables)

\begin{tabular}{|c|c|c|c|c|c|c|c|}
\hline Run & $\begin{array}{l}Q_{\text {water }} \\
\text { (Inlet) } \\
\text { [lpm] }\end{array}$ & $\begin{array}{l}<\mathbf{j}_{\mathbf{L}}> \\
\text { (inlet) } \\
{[\mathbf{m} / \mathbf{s}]}\end{array}$ & $\begin{array}{c}\mathbf{Q}_{\text {water }} \\
\text { Horizontal } \\
\text { Branch } \\
{[\text { lpm] }}\end{array}$ & $\begin{array}{c}<\mathbf{j}_{\mathrm{L}>} \\
\text { Horizontal } \\
\text { Branch } \\
{[\mathbf{m} / \mathbf{s}]}\end{array}$ & $\begin{array}{c}\text { Qair } \\
\text { (Port 1) } \\
\text { [INpm] }\end{array}$ & $\begin{array}{c}<\mathbf{j}_{\mathrm{G}}> \\
(\text { Port 1) } \\
{[\mathrm{m} / \mathrm{s}]}\end{array}$ & $\begin{array}{c}<\alpha> \\
(\text { Port 1) } \\
{[\%]}\end{array}$ \\
\hline R1A & 253 & 1.98 & 169 & 1.32 & 19.5 & 0.15 & 8.93 \\
\hline R1B & 259 & 2.03 & 155 & 1.22 & 50.3 & 0.39 & 19.01 \\
\hline R2A & 427 & 3.35 & 162 & 1.27 & 39.8 & 0.31 & 10.25 \\
\hline R2B & 431 & 3.39 & 154 & 1.21 & 77.6 & 0.61 & 17.92 \\
\hline
\end{tabular}

Pressure and temperature values for complete characterization of flow conditions are listed in Table II. It should be noted that due to the complexity and fluctuations involving a phase split in a Tjunction, maximum deviations of 10 mbar have been registered during tests in the pressures listed, which are considered in an acceptable range of accuracy. Table II also shows the corresponding gas fraction derived to horizontal and verticals sections upwards to T-junction $\left(Q_{\text {air }, H}\right.$ and $Q_{\text {air }, V}$, respectively). Presented values are obtained by referencing air flow rate measured at atmospheric conditions.

Table II. Experimental test conditions (Measured variables)

\begin{tabular}{ccccccccccc} 
& $\begin{array}{c}\text { P0 } \\
{[\mathbf{m B a r}]}\end{array}$ & $\begin{array}{c}\text { P1 } \\
{[\mathbf{m B a r}]}\end{array}$ & $\begin{array}{c}\text { PTI } \\
{[\mathbf{m B a r}]}\end{array}$ & $\begin{array}{c}\text { P2 } \\
{[\mathbf{m B a r}]}\end{array}$ & $\begin{array}{c}\text { PTO } \\
{[\mathbf{m B a r}]}\end{array}$ & $\begin{array}{c}\text { P3 } \\
{[\mathbf{m B a r}]}\end{array}$ & $\begin{array}{c}\text { Twater } \\
{\left[{ }^{\circ} \mathbf{C}\right]}\end{array}$ & $\begin{array}{c}\text { Tair } \\
{\left[{ }^{\circ} \mathbf{C}\right]}\end{array}$ & $\begin{array}{c}\mathbf{Q}_{\text {air,H }} \\
{[\%]}\end{array}$ & $\begin{array}{c}\mathbf{Q}_{\text {air,v }} \\
{[\% \mathbf{\%}]}\end{array}$ \\
\hline R1A & 544.27 & 430.13 & 234.48 & 268.42 & 235.66 & 86.63 & 20.44 & 19.50 & 69.47 & 29.79 \\
R1B & 513.16 & 405.10 & 213.08 & 254.48 & 228.86 & 83.72 & 20.13 & 19.13 & 72.60 & 29.89 \\
R2A & 572.78 & 444.60 & 226.35 & 263.42 & 250.99 & 99.50 & 20.38 & 19.20 & 46.78 & 49.74 \\
R2B & 544.28 & 426.64 & 210.17 & 251.17 & 244.98 & 97.81 & 20.37 & 19.09 & 49.87 & 49.01
\end{tabular}

All four experimental conditions have been repeated for each angle variation of the rotating section in the horizontal branch (7 rotations). Therefore, experimental results corresponding to the measurement ports located at vertical sections (Port 1 and Port 3) consist in the mean and the standard deviation.

Figure 6 shows photographs by using a conventional DSLR camera (Nikon D5000) of the T-junction for the test conditions. It can be noticed the existence of two vortices. First one is located at the entrance to the horizontal branch. The second right at the intersection, but on the opposite side to the horizontal section. These two vortices appear very clearly in the photographs as bubbles tend to get entrapped by them. The results in Table II indicate a better separation efficiency for lower liquid flow rates (R1A and R1B). In these photographs, this fact can be understood in terms of the vortex structure. The vortex in the horizontal branch grows with the inlet liquid velocity, so that the space 
left for the flow is smaller (this behavior is similar to the vena contracta in weirs). Also, the vortex at the junction tends to disappear and can hardly be noticed in the photos. Then, the flow is free to enter the vertical section.

R1A

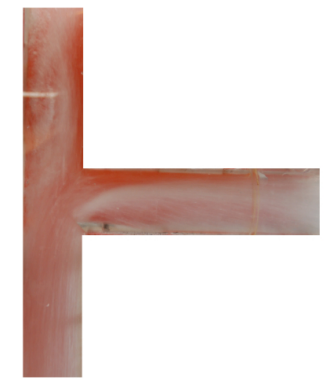

$\mathrm{R} 2 \mathrm{~A}$

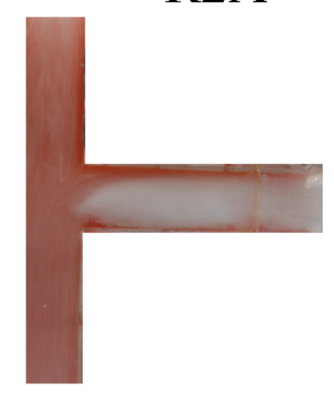

R1B

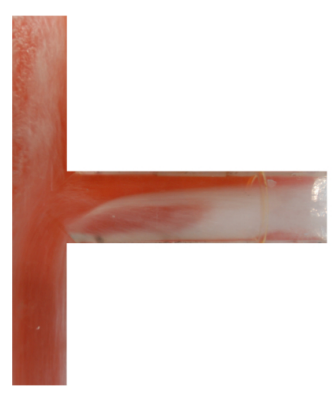

$\mathrm{R} 2 \mathrm{~B}$

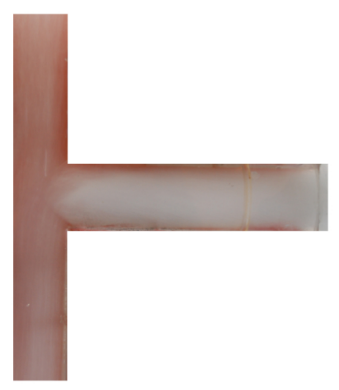

Figure 6. Changes in flow structure at T-junction for the test conditions under investigation.

\subsection{Void Fraction Measurements}

Figure 7 shows, from right to left, 3D, 2D and radial profiles of void fraction measurements at horizontal branch (Port 2) for all four conditions. For 3D and 2D representation, radial local values for 7 port rotations (from 0 to $180^{\circ}$ ) have been radially interpolated. The azimuthal directions corresponding to $0^{\circ}$ and $90^{\circ}$ denote the vertical axis and the horizontal axis of the pipe cross section, respectively. It can be observed a significant value of void fraction remains close to the bottom wall of the pipe, it stresses the fact that probe locations and port rotations must cover the bottom of the cross-section area for accurate local parameters measurement.

All void fraction distributions presented show a clear but different gradation in both flow conditions, as the disperse phase tend to move towards the top of the section due to buoyancy.

Despite the different inlet conditions, case R1A compared to R2A and case R1B compared to R2B present very close values for area-averaged void fraction and superficial liquid velocity, thus the small deviations could be explained by the mixing process at T-junction, which produces changes in the vortex geometry dependant of the inlet conditions: at higher air flow rates (R1B and R2B) the vortex axial length increases and approaches to measurement port, increasing the bubble dispersion in the measurement location. 

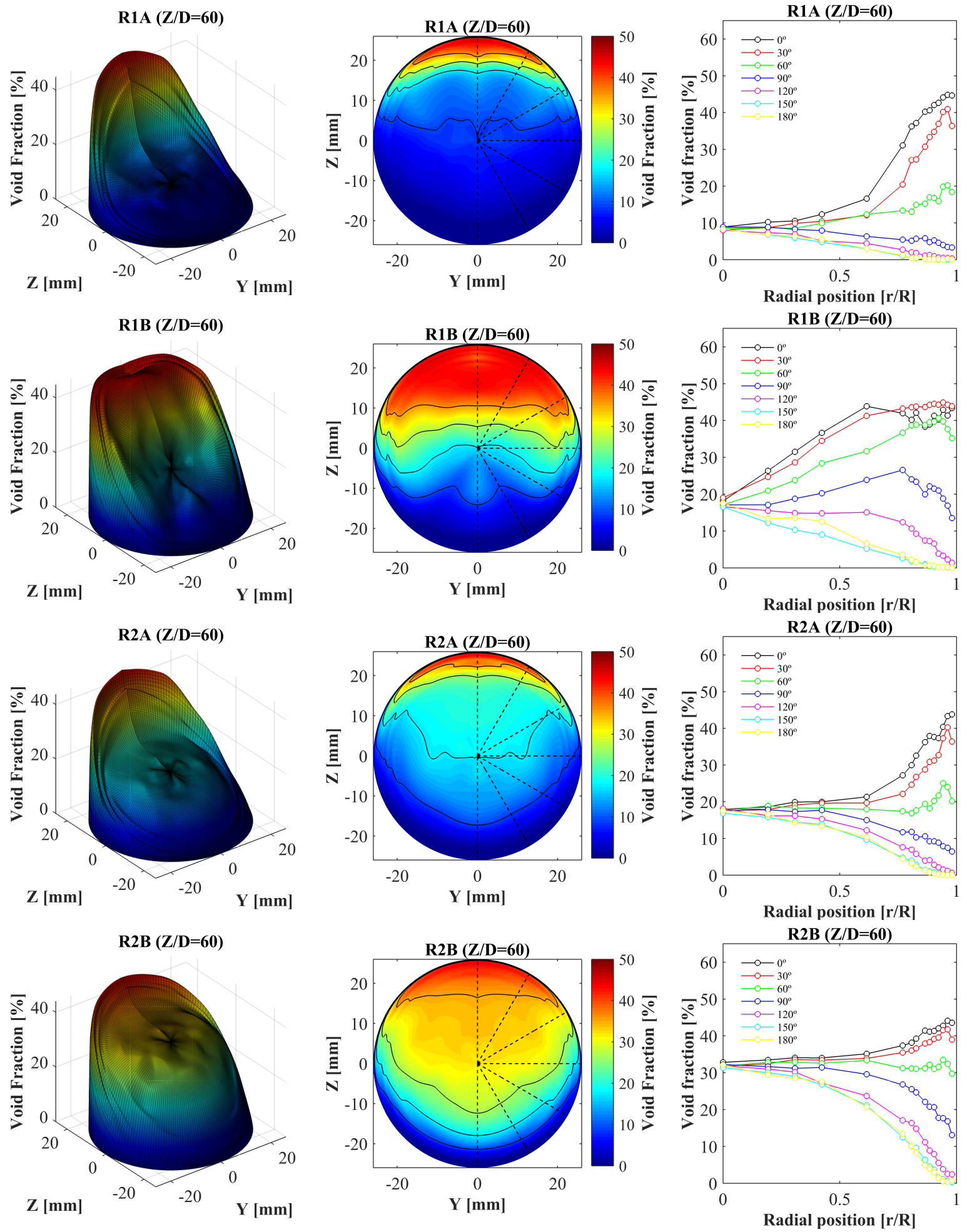

Figure 7. Void fraction measurements at Port 2.

Figure 8 shows the corresponding void fraction radial profiles at vertical section. It can be observed the characteristic wall-peak void profiles for all conditions at Port 3 and R1A and R2A at Port 1. Core-peak profiles are only achieved at port 1 for $\mathrm{R} 1 \mathrm{~B}$ and $\mathrm{R} 2 \mathrm{~B}$, corresponding to highest gas superficial velocities. Note that error bars are presented in the following figures for Port 1 and Port 3 
representing the standard deviation between measurement repetitions ( 7 repeated measurements corresponding to each angular position change in Port 2).
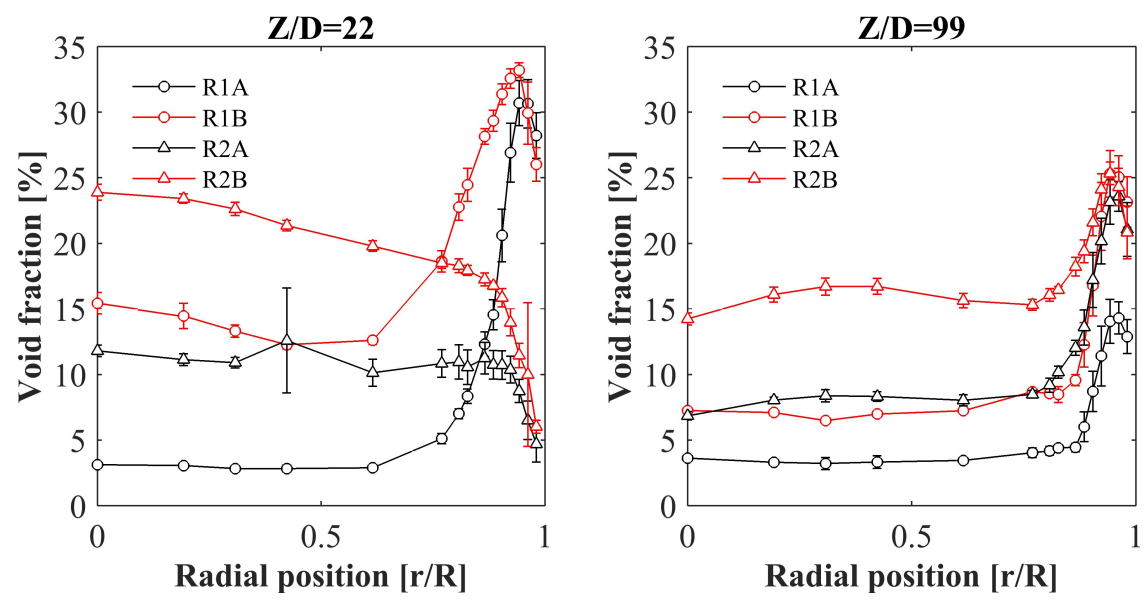

Figure 8. Void fraction radial profiles corresponding to vertical section measurement ports.

\subsection{Interfacial velocity}

Interfacial velocity in the flow direction measured in the horizontal branch (Port 2) is shown in Figure 9. It can be observed the lower velocity values near the top wall of the section, where the larger bubbles tend to coalesce are accumulated due buoyancy. Also the typical large velocity gradients near wall can be observed.

Note that if the number of bubbles detected and void fraction are below 200 and 2\%, respectively, at specific local measurement, interfacial velocity is set to zero, due to lack of samples for proper statistical interpretation. Thus it is assumed that there are no bubbles at this point or its influence for interfacial velocity is negligible. That's the reason for high velocity gradient present in all conditions. Nevertheless, the interfacial velocity is quite uniform in the core region of the pipe. Only a clear disturbance can be noticed for R1B condition near the top of the section, caused by the larger bubbles detected. 

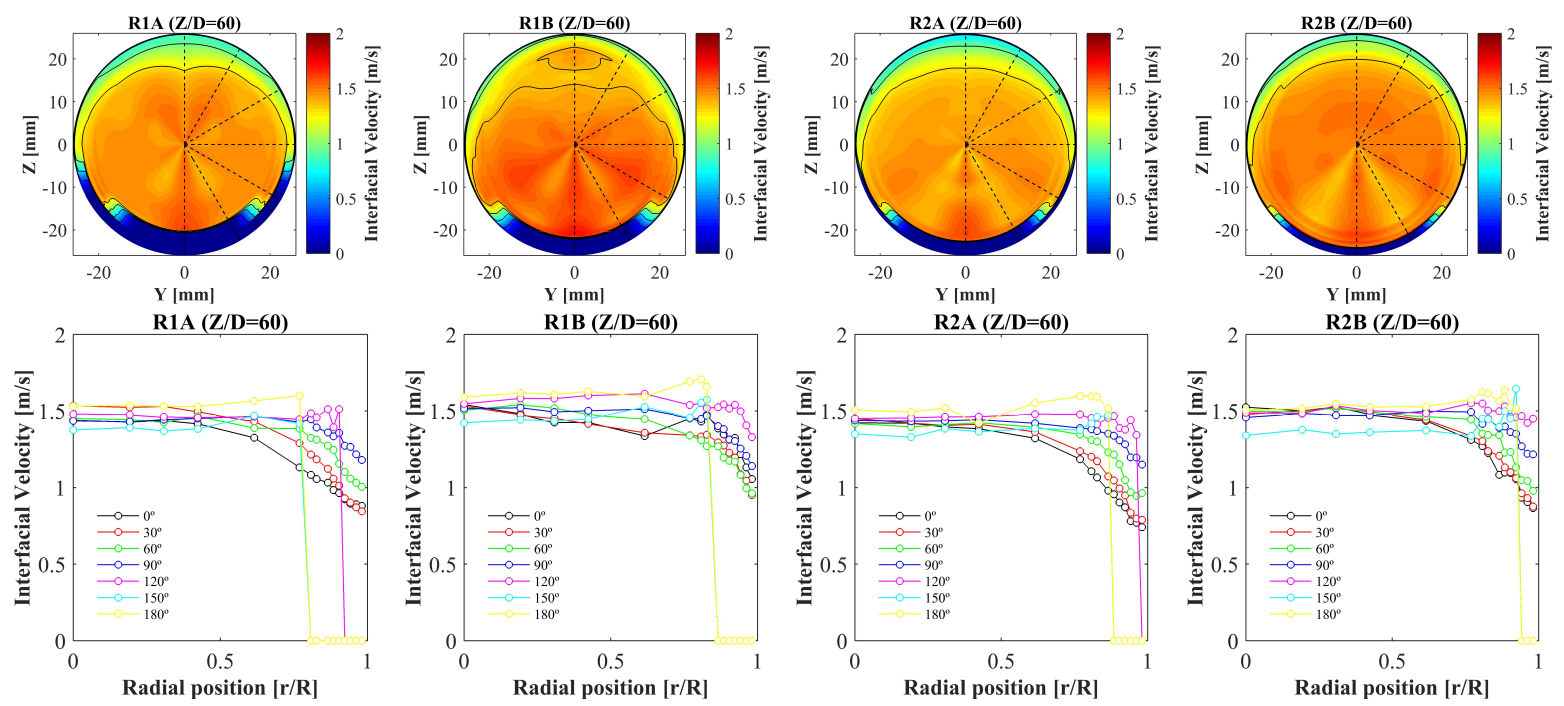

Figure 9. Interfacial velocity measurements at Port 2: cross sectional representation and radial values.

Figure 10 shows the corresponding interfacial velocity profiles at vertical section. The effect of liquid phase separation over interfacial velocity: a noticeable decrease in interfacial velocity between Port 1 and Port 3 is observed. For all experimental conditions, measurements in Port 3 compared to the corresponding cases at Port 1 show the typical bubble flattening effect over interfacial velocity profiles, characteristic of bubbly flow patterns with moderate liquid flow rates and low void fractions. As expected the velocity radial profiles show similar trend and increases with air flow-rate.
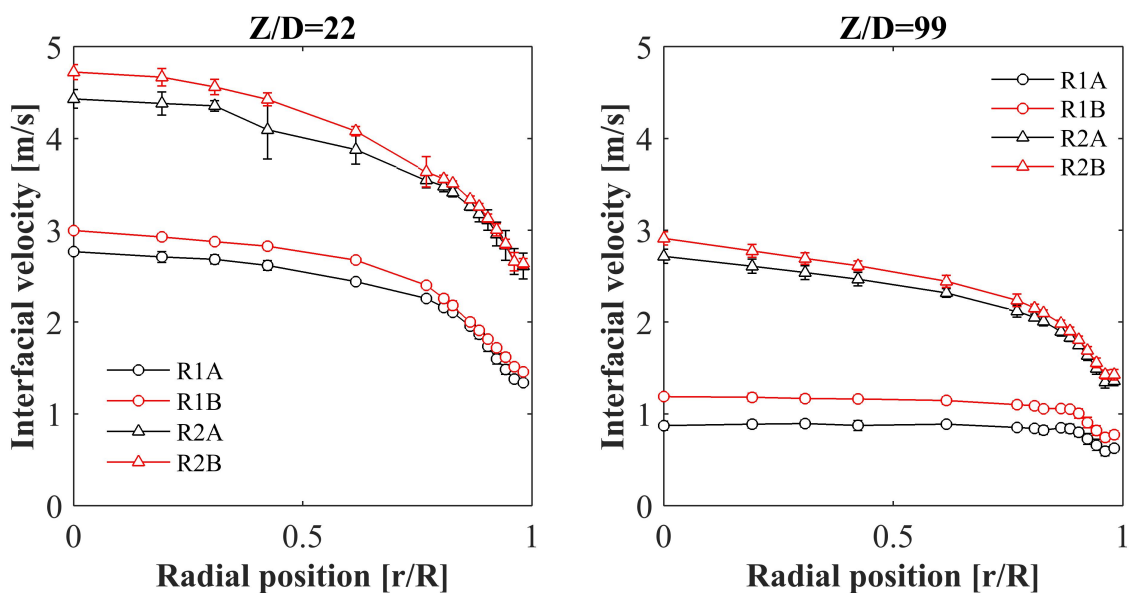

Figure 10. Interfacial velocity radial profiles corresponding to vertical section measurement ports.

\subsection{Chord Length}

Figure 11 depicts the distribution of mean chord lengths computed at the cross section. It shows a uniform and constant mean chord length value for cases R1A, R2A and R2B, which stress the fact that also bubble sizes are similar and with limited dependence to flow conditions. However, case R1B depicts a change in 
this trend. Larger bubbles are detected in the upper part of the pipe section, which explains the changes in the interfacial velocity at same zone, as stated in the previous section.
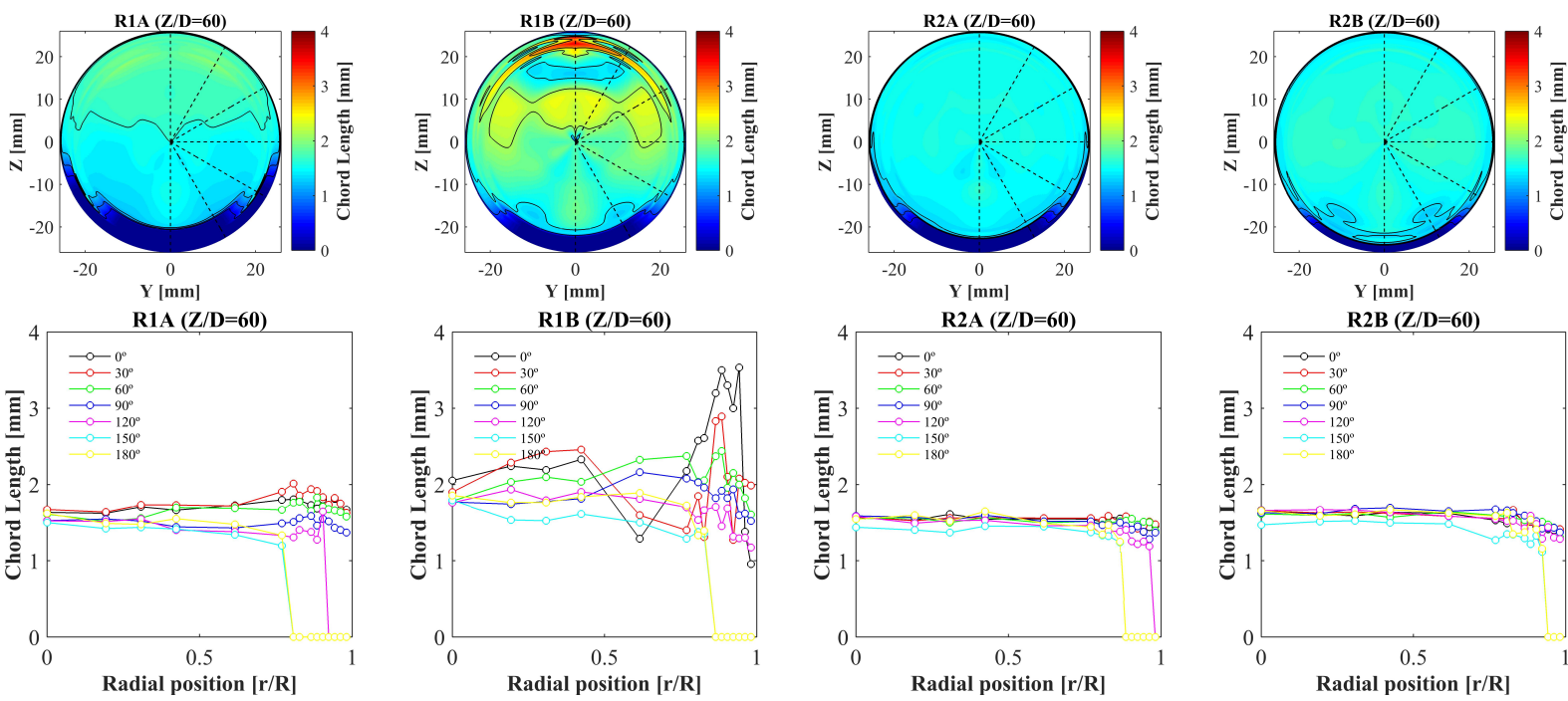

Figure 11. Mean chord length measurements at Port 2: cross sectional representation and radial values.

According to (Mandhane et al., 1974), this corresponds to transition zone between disperse flow and plug flow pattern. In this regime small bubble population is still dominant and causes limited, although noticeable, influence of the larger bubbles in the mean chord length. The higher large bubble detected rate in R1B condition is better depicted in Figure 12 (left), where a comparison between chord length PDF's for cases R1A and R1B is presented.
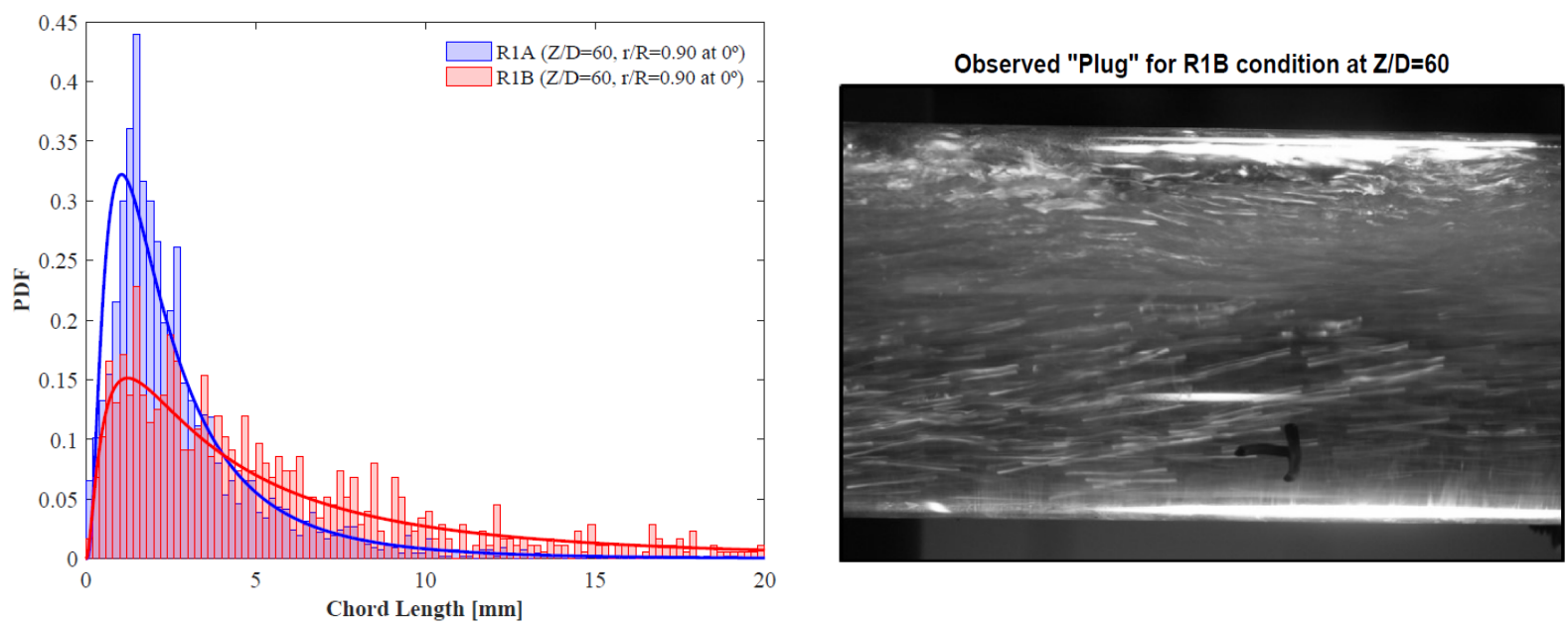

Figure 12. Chord length PDF's close to upper pipe wall at Port 2 (left) and confirmation image for measured large bubbles, at $\mathrm{X} / \mathrm{D}=10$ (right).

The radial variations of mean chord length at vertical section are presented in Figure 13. It can be seen that, for constant liquid superficial velocity, mean chord length increases at the center of the pipe with increasing gas superficial velocity. It can also be noted that for cases corresponding to wall-peak void distribution, mean chord length values presents a peak near the wall. This phenomena is well explained by 
(Kalkach-Navarro et al., 1993) from the fact that the presence of a wall limits the arc length at which the bubble is intercepted, thus the radial location of the peak is related to maximum bubble radius.

For high gas superficial velocity cases at Port 1 where void fraction shows a core-peak distribution (cases R1B and R2B), higher mean chord length values can be found, as larger bubbles tend to move towards the center of the pipe due to lift force (Tomiyama et al., 2002).
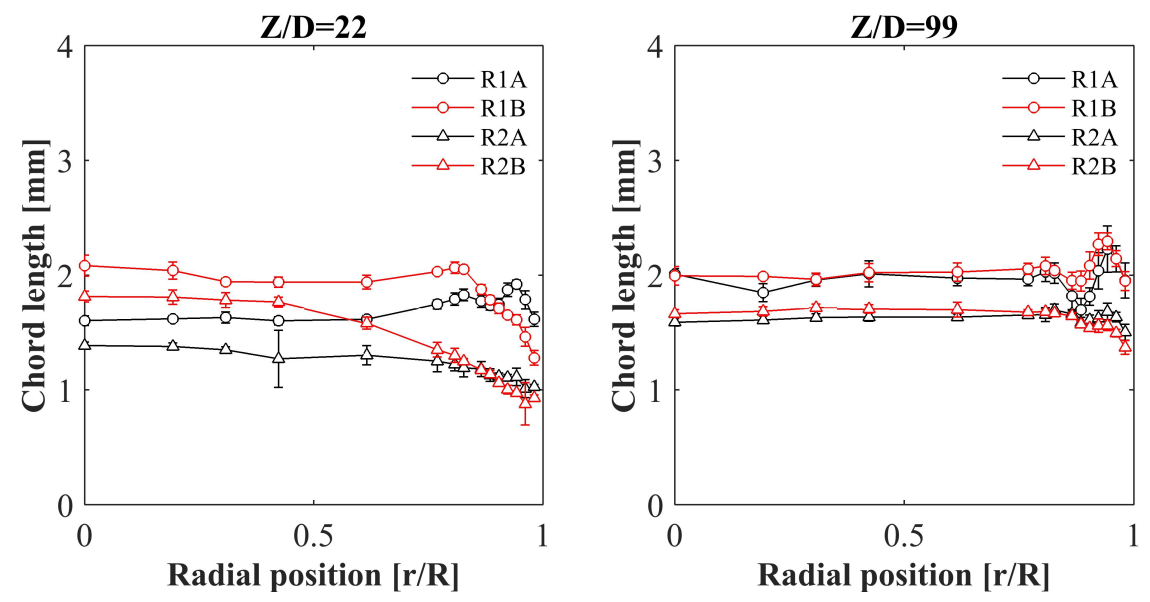

Figure 13. Mean chord length radial profiles corresponding to vertical section measurement ports.

\subsection{Interfacial Area Concentration (IAC)}

Figure 14 shows the different IAC distribution in horizontal branch Port 1 cross-section. The IAC distributions obtained are very similar to the ones obtained for void fraction. This is expected for bubbly flow conditions where the surface area and volume of a bubble are linearly related. Lower values of IAC at top section in R1B stress the fact that larger bubbles are formed due coalescence processes, with typical lower contribution to IAC rather than small bubbles. 

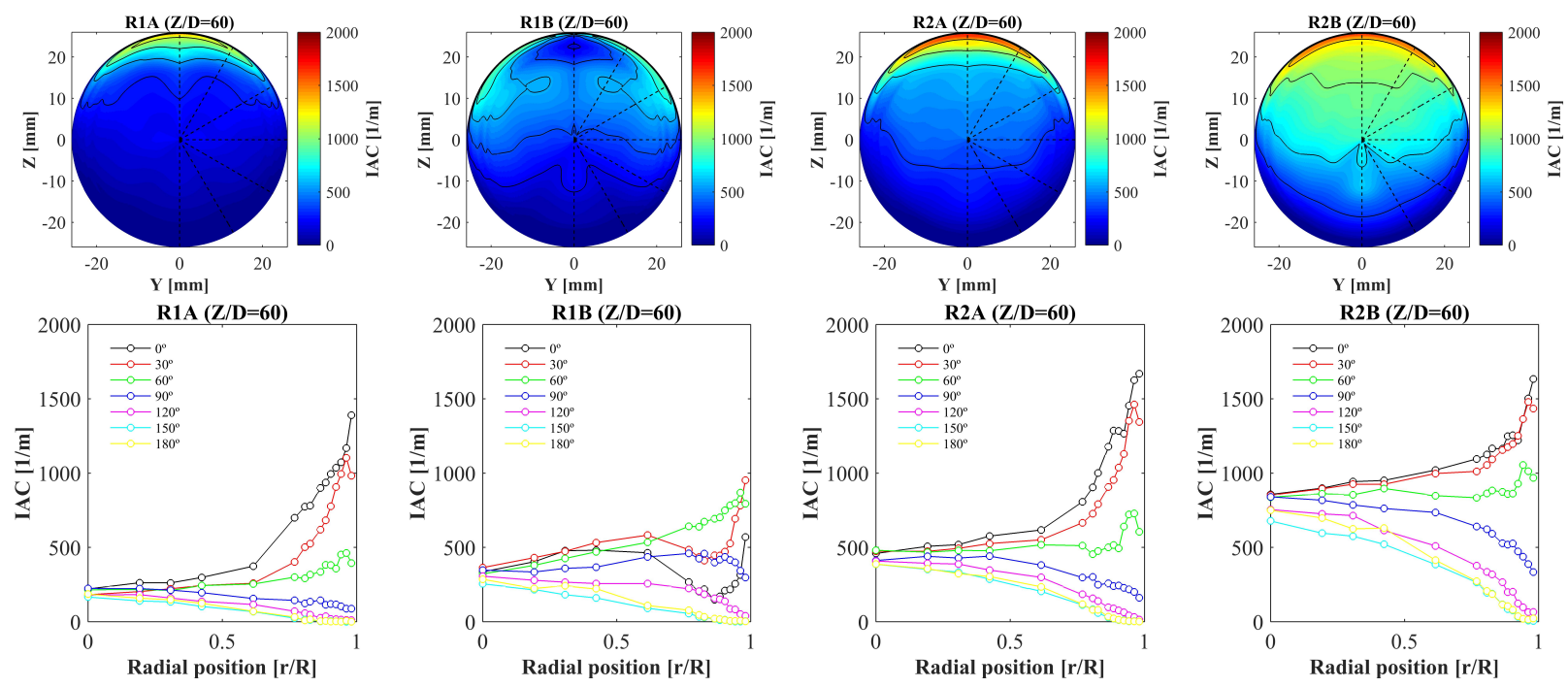

Figure 14. IAC measurements at Port 2: cross sectional representation and radial values.

Figure 15 shows the IAC profiles in vertical sections. As stated for Port 2 measurements, IAC profiles at vertical section are also similar to its corresponding void fraction profiles. As expected, wall-peaked profiles are obtained as the major contribution of IAC is produced where the bubble density (or void fraction) is higher (Figure 8). Cases R1A and R1B presents lower values of local IAC in the core region, caused by the larger bubbles detected in this region (Figure 12), which is also a usual characteristic of core-peak void profiles (Figure ).
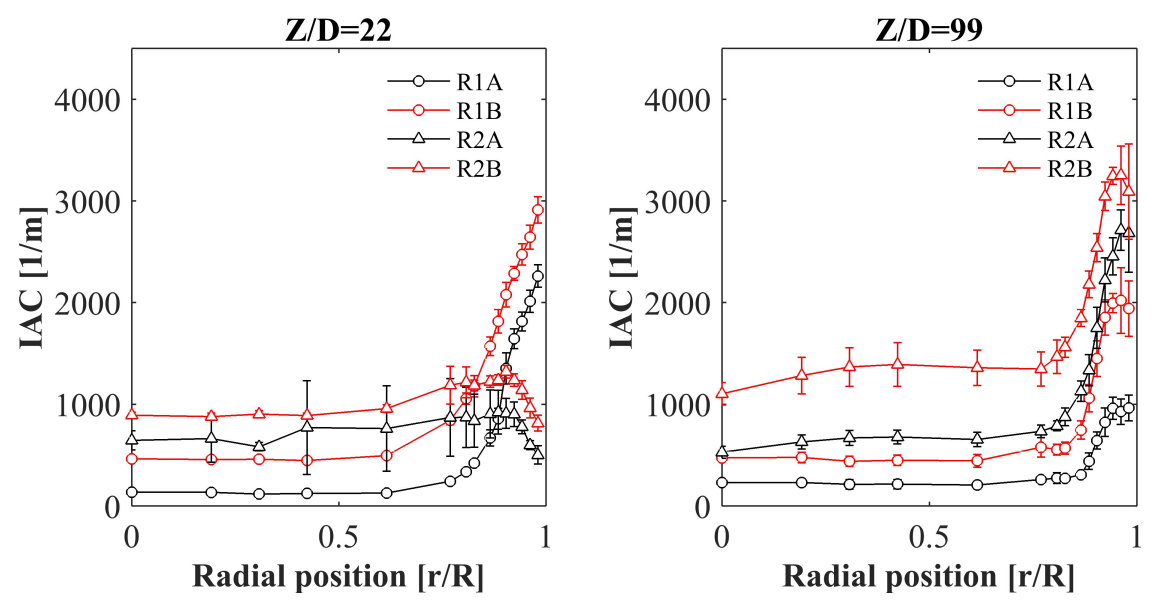

Figure 15. IAC radial profiles corresponding to vertical section measurement ports.

As IAC is a key parameter governing energy, mass and momentum transfer, the evaluation of local IAC provides useful information beyond area-averaged values. In the case of a T-junction, it could be very important to study thermal stresses (local IAC and void fraction), fatigue and pipe erosion (local IAC and bubble distribution) and chemical reactions (local IAC distribution)

\subsection{Bubble Frequency}


In Figure 16, top of the cross section for condition R1B shows a decreasing value of bubble frequency compared with the surroundings. This fact suggests, as Fig.8, that coalescing processes are happening, confirmed by intermittent flow observed at this condition.

Bubble frequency distributions corresponding to Port 2 measurements in Figure 6 show similar patterns to their counterparts for the case of void fraction and IAC, as expected. Again, top of the cross section for condition R1B shows a decreasing value of bubble frequency compared with the surroundings, which stress the fact of the existence of larger bubbles.
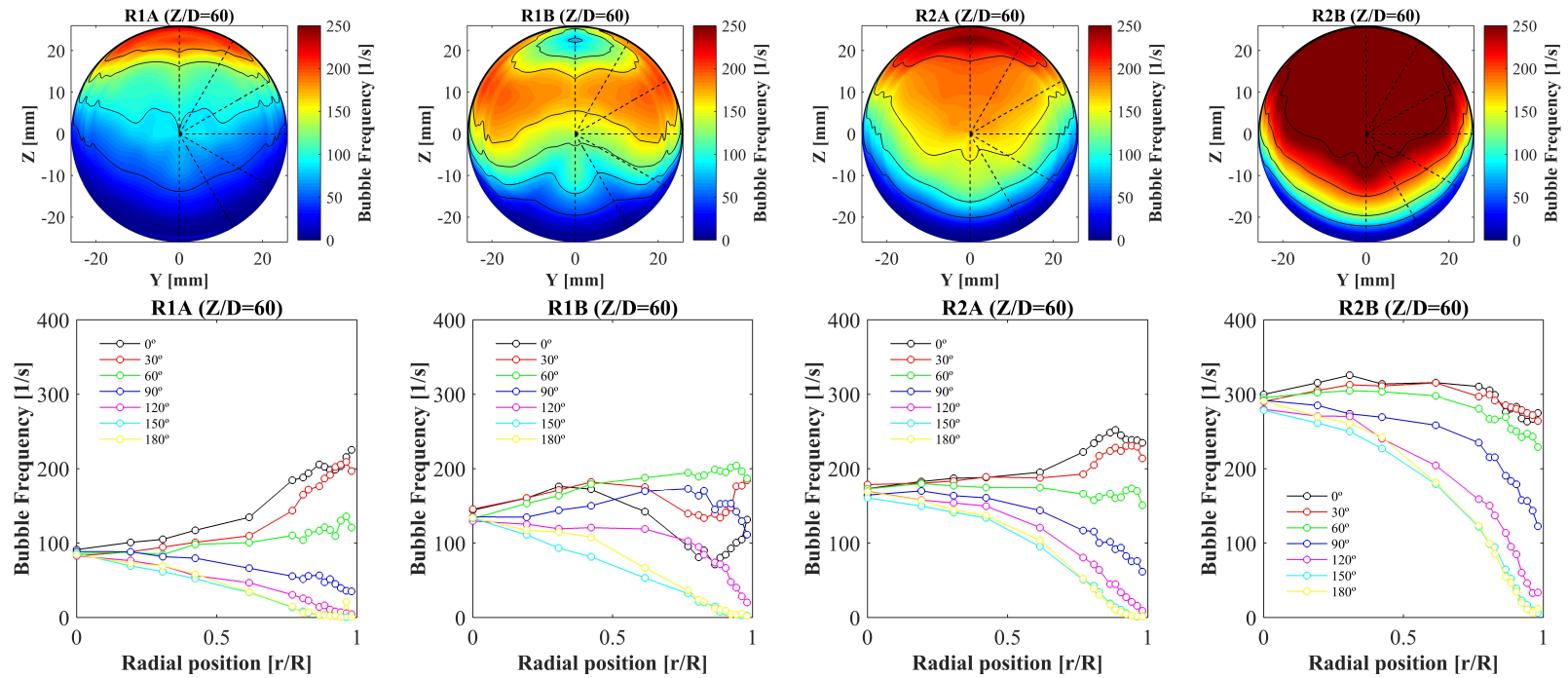

Figure 16. Bubble frequency measurements at Port 2: cross sectional representation and radial values.

As expected for bubbly flow conditions, Figure 17 shows that increasing gas fraction at constant liquid flow rate also increases local bubble frequency, with similar radial profiles as void fraction and IAC.
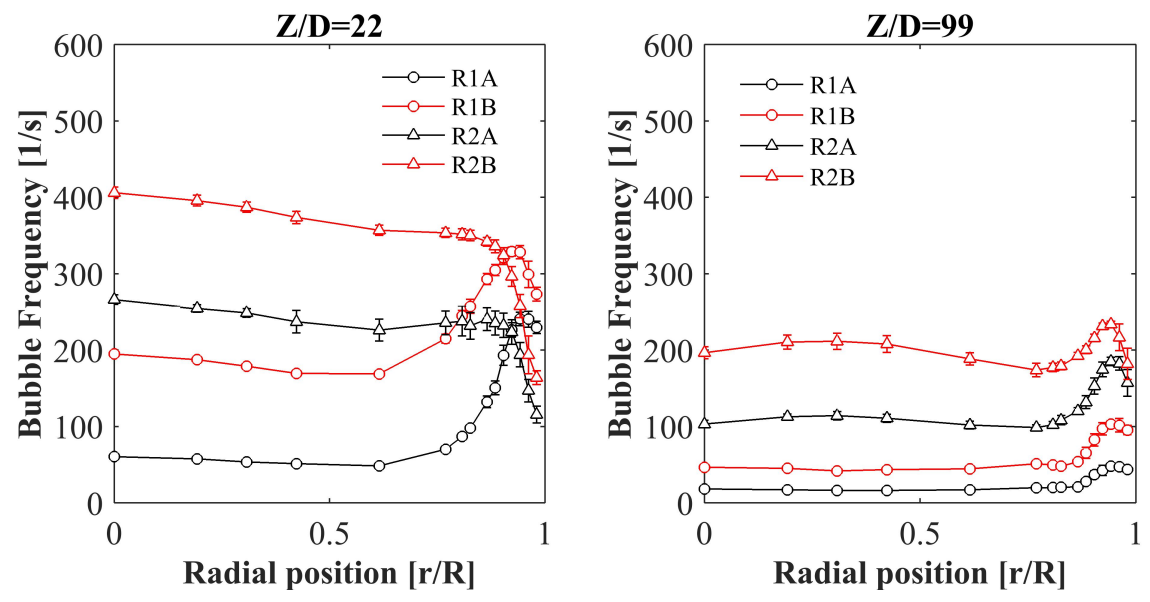

Figure 17. Bubble frequency radial profiles corresponding to vertical section measurement ports.

\subsection{Disperse phase area-averaged local flow parameters and probe measurements validation}

As summary, Table III presents the area-averaged local flow parameters for all tested conditions: void fraction, $\langle\alpha\rangle$, axial interfacial velocity, $\left\langle V_{b}\right\rangle$, interfacial area concentration, $\langle I A C\rangle$, mean chord length, 
$<$ Chord $>$, and bubble frequency, $\left\langle N_{f}>\right.$. These results are suitable for the validation of collapsed onedimensional codes. Also, it can be noticed an interesting fact: flow speed through the horizontal branch is remains practically constant for all the conditions. There is no influence when the inlet liquid flow rate is changed, and there is a slight contribution when void is increased. This suggests a saturation behavior of the T-junction as a separator. This is reinforced by the measurements of void fraction. The void at the top port tends to decrease with respect to the inlet one for low speed conditions, but it remains practically the same for higher speed conditions. Thus, air tends to flow vertically.

Table III. Area-averaged main local flow parameters.

\begin{tabular}{lccccc} 
& $\begin{array}{c}<\boldsymbol{\alpha}> \\
{[\mathbf{\%}]}\end{array}$ & $\begin{array}{c}\left\langle\mathbf{V}_{\mathbf{b}}>\right. \\
{[\mathbf{m} / \mathbf{s}]}\end{array}$ & $\begin{array}{c}<\text { IAC } \\
{[\mathbf{1} / \mathbf{m}]}\end{array}$ & $\begin{array}{c}<\text { Chord } \\
{[\mathbf{m m}]}\end{array}$ & $\begin{array}{c}\left.<\mathbf{N}_{\mathbf{f}}\right\rangle \\
{[\mathbf{1} / \mathbf{s}]}\end{array}$ \\
\cline { 2 - 6 } R1A (Z/D=22) & 8.93 & 2.17 & 277 & 1.66 & 96.0 \\
R1A (Z/D=60) & 9.81 & 1.19 & 226 & 1.36 & 76.5 \\
R1A (Z/D=99) & 5.25 & 0.81 & 200 & 1.92 & 22.7 \\
\hline R1B (Z/D=22) & 19.0 & 2.34 & 508 & 1.86 & 218 \\
R1B (Z/D=60) & 22.0 & 1.31 & 337 & 1.72 & 121 \\
R1B (Z/D=99) & 10.2 & 1.05 & 413 & 2.00 & 55.2 \\
\hline R2A (Z/D=22) & 10.3 & 3.56 & 337 & 1.21 & 222 \\
R2A (Z/D=60) & 14.8 & 1.24 & 409 & 1.37 & 133 \\
R2A (Z/D=99) & 10.9 & 2.08 & 487 & 1.60 & 117 \\
\hline R2B (Z/D=22) & 17.9 & 3.71 & 459 & 1.39 & 334 \\
R2B (Z/D=60) & 25.5 & 1.34 & 665 & 1.47 & 222 \\
R2B (Z/D=99) & 17.2 & 2.19 & 790 & 1.62 & 193 \\
\hline
\end{tabular}

In order to verify the accuracy of the local four sensor probe measurements, the area averaged quantities, obtained by integrating the local flow parameters over the flow, have been compared with those obtained by the rotameter (horizontal branch) and by the flow controller (inlet) as depicted in Figure 18. For vertical section measurement locations, cross-sectional averaged gas superficial velocity from probes $\left(J_{g, P R O B E S}\right)$ is calculated based on radial void fraction $(\alpha)$ and axial local interfacial velocity $\left(V_{z)}\right.$ measured in the experiments by integration over the cross-section (Eq. 9).

$$
J_{g, P R O B E S}=\frac{2 \pi}{A} \int_{0}^{R} \alpha(r) V_{z}(r) r d r
$$

For Port 2 measurements (horizontal section), cross-sectional averaged gas superficial velocity is computed as the summation of $\alpha$ and local axial velocity $\left(V_{x}\right)$ resampled over full cross-section. 


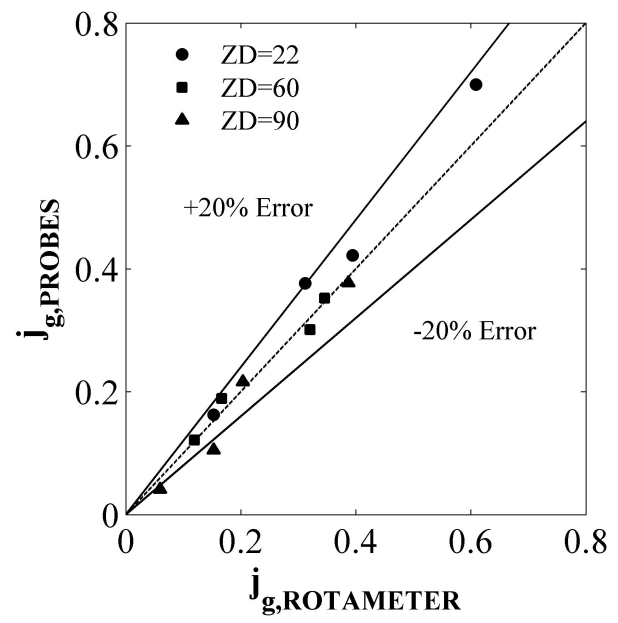

Figure 18. Air flow rate comparison. Values obtained with flow meters and four sensor probes.

The averaged and maximum relative deviations of air superficial velocity, between the four-sensor probes and rotameter or flow controller, are $7.8 \%$ and $22.1 \%$, so a very good agreement has been achieved for all flow conditions, as can it can be compared in the literature for similar works involving two-phase flow area-averaged based validations (Shen and Nakamura, 2013).

\subsection{Liquid Velocity Measurements in the T-junction}

In order to quantify the liquid velocity in the splitting region, six measurements are performed around the T-junction center for each condition by using LDA. The raw velocity signals provide the components of the velocity in ZX plane, the estimation of turbulent intensity (TI) and turbulent kinetic energy (TKE).

As demonstrated by (Mudde et al., 1997), LDA measurements using back-scattering modality tends to prioritize liquid phase (even without seeding particles) and provides suitable results up to $\sim 25 \%$ void fraction, therefore no extra raw-signal post-processing has been used to account the presence of bubbles.

$\mathrm{TI}$ in the $\mathrm{Z}$ and $\mathrm{X}$ directions is computed from the average velocity of the liquid $\left(U_{l}\right)$ and standard deviation $(u$ ') of the LDA velocity data:

$$
T I_{X}=\frac{u_{X}^{\prime}}{U_{l, X}}, T I_{Z}=\frac{u_{Z}^{\prime}}{U_{l, Z}}
$$

TKE has been computed as Eq. (X) assuming similar velocity fluctuations in the $\mathrm{X}$ and $\mathrm{Y}$ directions $\left(u_{X}^{\prime} \approx u_{Y}^{\prime}\right)$, as long as only two directional measurements (two laser lines) are available.

$$
T K E=\frac{1}{2}\left(\left(u_{X}^{\prime}\right)^{2}+\left(u_{Y}^{\prime}\right)^{2}+\left(u_{Z}^{\prime}\right)^{2}\right) \approx \frac{1}{2}\left(2\left(u_{X}^{\prime}\right)^{2}+\left(u_{Z}^{\prime}\right)^{2}\right)
$$

Figure 19 shows the air distribution in the T-junction during the phase separation process and liquid velocity vectors in the measured positions (A, B, C, D, E and F), providing information about the split process (noticeable velocity decay from $\mathrm{D}$ to $\mathrm{B}$ ) and the evolution of the liquid core (vector orientation and magnitude of A, C, E and F). The increment of injected gas fraction between experimental conditions has a noticeable influence over liquid parameters although the air mass flow rate diverted remains constant as stated previously. 
Values of local liquid parameters mentioned above are shown in Table III. The TI values highlight the complexity of the splitting process, as it is extremely turbulent in the horizontal branch direction. Those extreme IT values can be explained by the high fluctuations observed in the liquid core.
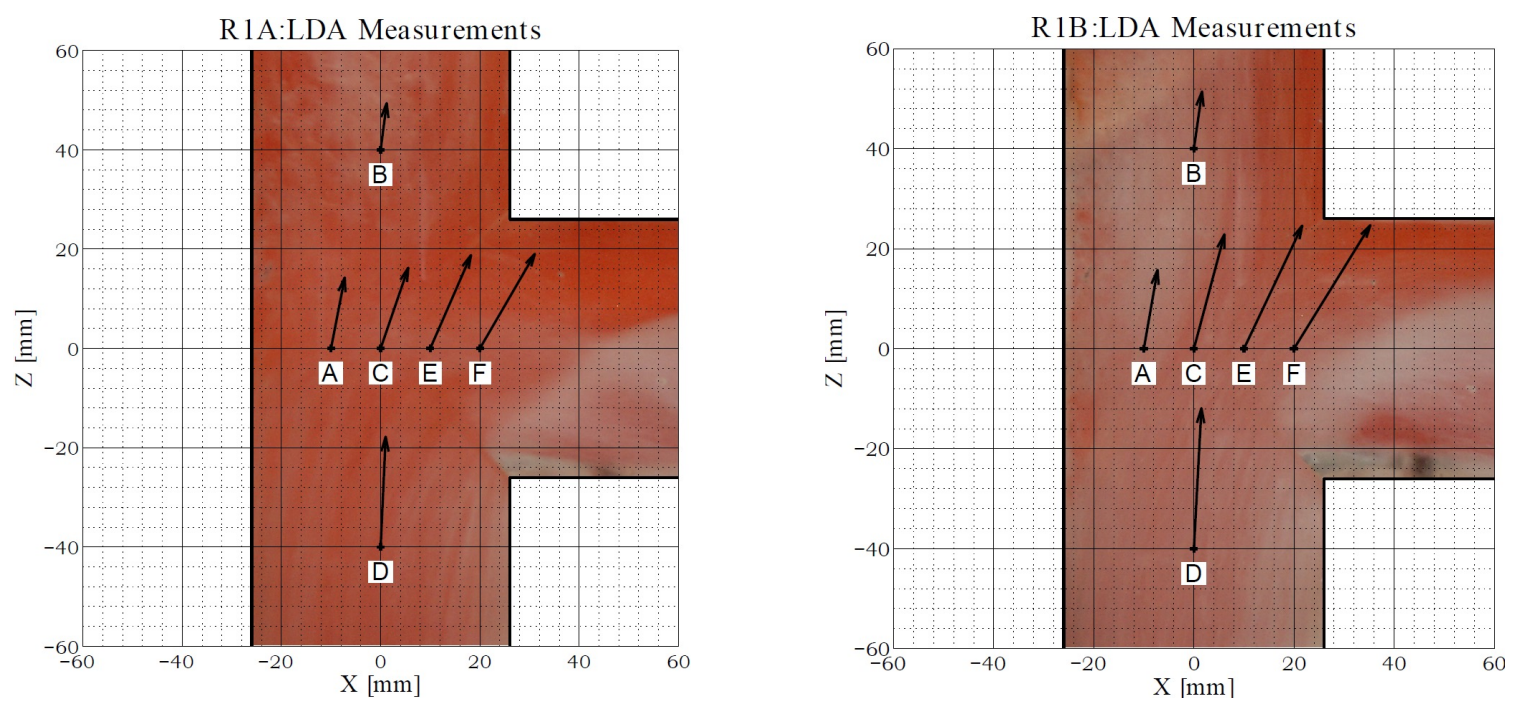

Figure 19. Liquid velocity measurements inside T-junction, velocity modulus scaled 1:100.

Table III. Liquid local flow parameters

\begin{tabular}{|c|c|c|c|c|c|}
\hline Location & $\mathbf{V}_{x}[\mathrm{~m} / \mathrm{s}]$ & $\mathrm{V}_{\mathrm{z}}[\mathrm{m} / \mathrm{s}]$ & $\mathbf{T I}_{\mathbf{x}}[\%]$ & $\mathrm{TI}_{\mathrm{z}}[\%]$ & TKE $\left[\mathrm{m}^{2} / \mathrm{s}^{2}\right]$ \\
\hline A & 0.30 & 1.58 & 532 & 24.1 & $1.35 \mathrm{E}+00$ \\
\hline B & 0.13 & 1.04 & 199 & 57.7 & $2.13 \mathrm{E}-01$ \\
\hline $\mathrm{C}$ & 0.61 & 1.80 & 16.5 & 7.14 & $1.33 \mathrm{E}-02$ \\
\hline $\mathrm{D}$ & 0.11 & 2.46 & 49.0 & 3.22 & $4.59 \mathrm{E}-03$ \\
\hline $\mathrm{E}$ & 0.90 & 2.08 & 7.22 & 6.30 & $1.07 \mathrm{E}-02$ \\
\hline $\mathrm{F}$ & 1.20 & 2.11 & 5.96 & 5.41 & 0.01 \\
\hline A & 0.30 & 1.74 & 105 & 37.9 & $2.67 \mathrm{E}-01$ \\
\hline B & 0.18 & 1.27 & 197 & 60.9 & 3.63E-01 \\
\hline $\mathrm{C}$ & 0.67 & 2.53 & 22.7 & 7.79 & $3.10 \mathrm{E}-02$ \\
\hline $\mathrm{D}$ & 0.16 & 3.11 & 99.1 & 6.14 & $3.08 \mathrm{E}-02$ \\
\hline $\mathrm{E}$ & 1.29 & 2.73 & 37.4 & 20.6 & $2.73 \mathrm{E}-01$ \\
\hline \multirow{7}{*}{$\begin{array}{l}\mathrm{F} \\
\mathrm{A} \\
\mathrm{B} \\
\mathrm{C} \\
\mathrm{D} \\
\mathrm{E} \\
\mathrm{F}\end{array}$} & 1.69 & 2.74 & 37.4 & 26.7 & 0.47 \\
\hline & - & - & - & - & - \\
\hline & 0.21 & 0.69 & 111 & 90.7 & $2.24 \mathrm{E}-03$ \\
\hline & 0.58 & 1.76 & 19.0 & 9.96 & $2.14 \mathrm{E}-04$ \\
\hline & 0.11 & 2.31 & 59.0 & 3.91 & $6.08 \mathrm{E}-05$ \\
\hline & 0.97 & 1.71 & 9.37 & 98.3 & $1.41 \mathrm{E}-02$ \\
\hline & 1.33 & 1.72 & 6.06 & 99.7 & 0.01 \\
\hline
\end{tabular}

Values for turbulence at D show a strong agreement with the turbulence computation in upward singlephase fully developed pipe flow at the core (Wilcox, 1998).

Generally, an increase in turbulent kinetic energy is noticed as the void fraction increases thanks to the bubble induce turbulence mechanism. Location $\mathrm{F}$ is placed right at the border of the vortex entering the 
horizontal branch. The huge values for the turbulence field indicates strong oscillations of the vortex borders, so this point can be either in or out the vortex as time evolves. This non-steady nature of the vortex was observed during the experiments, and should be taken into account for the simulation of the system with CFD codes.

Finally, a huge difference of turbulence kinetic energy at point A between cases R1A and R1B is noticed. For low gas conditions the vortex is located in A diverting the flow towards the horizontal branch. For high liquid speed this vortex moves upwards, so the flow keeps flowing mainly through the vertical section.

\section{CONCLUSIONS}

Methodology involving four-sensor conductivity probes was applied to the measurement of various key local parameters for an upward two-phase flow system with a splitting T-junction. Local parameters of both gas and liquid phases were obtained, including the void fraction, interfacial velocity, and IAC and bubble frequency for disperse phase, and liquid velocity, turbulent intensity and turbulent kinetic energy for the continuous phase.

The presented results indicate that the T-junctions act better as gas-liquid separators for low liquid inlet velocities, and as flow diverters for high liquid speed conditions. This trend seems to be caused by the vortex structure within the T-junction. The vortex in the horizontal branch grows with liquid speed and impedes the entrance of flow through this branch. At the same time, the vortex at the T-junction itself tends to disappear when liquid flow rate increases, further increasing the trend of the flow to keep moving along the vertical direction.

In addition, the measurements for void fraction and axial interfacial velocity component were checked against the void fraction measurements using the superficial gas velocity measurement using gas flow meters at two measurement ports. A remarkable agreement was found, considering the complexity of performing simultaneous measurements in two very different flow patterns, horizontal and vertical upward flow, which flow structure are influenced by the phase segregation in a T-junction.

As future work, further experimental work should be conducted involving local measurements performed across the entire horizontal branch in order to obtain the local parameters evolution with the horizontal flow development and also local measurements in both phases inside the T-junction, in order to fully characterize the complex flow behavior in this element. In the same way, regarding vertical section, additional measurements close to T-junction entrance and vertical exit would provide useful data to determine its influence on the vertical two-phase flow development. Also, liquid inlet velocities should be changed smoothly for a better understanding of the vortex structure development and the flow diversion as well as measurements.

\section{ACKNOWLEDGMENTS}

This work has been founded by the project ENE2013-48565-C2-2-P, financed by the Ministerio de Economía y Competitividad (Spain). G. Monrós-Andreu gratefully acknowledges a FPI-2011 predoctoral scholarship, from Ministerio de Economía y Competitividad.

\section{REFERENCES}

Azzopardi, B., 1982. The effect of flow patterns on two-phase flow in a T junction. Int. J. Multiph. Flow $8,491-507$.

Azzopardi, B.J., 1984. The effect of the side arm diameter on the two-phase flow split at a "T" junction. 
Int. J. Multiph. Flow 10, 509-512. doi:10.1016/0301-9322(84)90059-4

Azzopardi, B.J., Colman, D.A., Nicholson, D., 2002. PLANT APPLICATION OF A T-JUNCTION. Chem. Eng. Res. Des. 80, 87-96.

Barrau, E., Poupot, C., Cartellier, A., 1999. Single and double optical probes in air-water two-phase flows : real time signal processing and sensor performance. Int. J. Multiph. Flow 25, 229-256.

Cartellier, a., 1999. Post-treatment for phase detection probes in non uniform two-phase flows. Int. J. Multiph. Flow 25, 201-228. doi:10.1016/S0301-9322(98)00041-X

Charron, Y., Whalley, P.B., 1995. Gas-liquid annular flow at a vertical tee junction - Part I. Flow separation 21, 569-589.

Dias, S.G., Franc, F.A., Rosa, E.S., 2000. Statistical method to calculate local interfacial variables in twophase bubbly fows using intrusive crossing probes. Int. J. Multiph. Flow 26, 1797-1830.

Euh, D.J., Yun, B.J., Song, C.H., Kwon, T.S., Chung, M.K., Lee, U.C., 2001. Development of the fivesensor conductivity probe method for the measurement of the interfacial area concentration. Nucl. Eng. Des. 205, 35-51. doi:10.1016/S0029-5493(00)00376-9

$\mathrm{Fu}, \mathrm{X} ., 2$ 2001. Interfacial area measurement and transport modeling in air-water two- phase flow. PhD. Thesis. Purdue University, School of Nuclear Engineering. doi:10.16953/deusbed.74839

Guet, S., Fortunati, R. V, Mudde, R.F., Ooms, G., 2003. Bubble Velocity and Size Measurement with a Four-Point Optical Fiber Probe Se. Part. Part. Syst. Charact. 20, 219-230.

Issa, R.I., Oliveira, P.J., 1994. Numerical prediction of phase separation in two-phase flow through Tjunctions 23, 347-372.

Kalkach-Navarro, S., Lahey, R.T., Drew, D. a, Meyder, R., 1993. Interfacial Area Density, Mean Radius and Number Density-Measurements in Bubbly 2-Phase Flow. Nucl. Eng. Des. 142, 341-351. doi:10.1016/0029-5493(93)90212-R

Kataoka, I., Ishii, M., Serizawa, A., 1986. Local formulation and measurements of interfacial area concentration in two-phase flow. Int. J. Multiph. Flow 12, 505-529. doi:10.1016/03019322(86)90057-1

Kataoka, I., Serizawa, A., 1990. Interfacial area concentration in bubbly flow. Nucl. Eng. Des. 120, 163180. doi:10.1016/0029-5493(90)90370-D

Kim, S., Fu, X.Y., Wang, X., Ishii, M., 2001. Study on interfacial structures in slug flows using a miniaturized four-sensor conductivity probe. Nucl. Eng. Des. 204, 45-55. doi:10.1016/S00295493(00)00312-5

Kim, S., Fu, X.Y., Wang, X., Ishii, M., 2000. Development of the miniaturized four-sensor conductivity probe and the signal processing scheme. Int. J. Heat Mass Transf. 43, 4101-4118. doi:10.1016/S0017-9310(00)00046-6

Le Corre, J.-M., Ishii, M., 2002. Numerical evaluation and correction method for multi-sensor probe measurement techniques in two-phase bubbly flow. Nucl. Eng. Des. 216, 221-238. doi:10.1016/S0029-5493(02)00130-9

Liu, T.J., Bankoff, S.G., 1993. Structure of air-water bubbly flow in a vertical pipe-II. Void fraction, bubble velocity and bubble size distribution. Int. J. Heat Mass Transf. 36, 1061-1072. doi:10.1016/S0017-9310(05)80290-X

Mandhane, J.M., Gregory, G.A., Aziz, K., 1974. A flow pattern map for gas-liquid flow in horizontal pipes. Int. J. Multiph. Flow 1, 537-553.

Mudde, R.F., Groen, J.S., Van Der Akker, H.E.A., 1997. Liquid velocity field in a bubble column: LDA experiments. Chem. Eng. Res. Des. 52, 4212-4224.

Pradhan, S.R., Mishra, R., Ubbi, K., Asim, T., 2013. Sensors and Actuators A : Physical Optimal design of a four-sensor probe system to measure the flow properties of the dispersed phase in bubbly air water multiphase flows. Sensors Actuators A. Phys. 201, 10-22. doi:10.1016/j.sna.2013.06.014

Revankar, S.T., Ishii, M., 1992. Local interfacial area measurement in bubbly flow. Int. J. Heat Mass Transf. 35, 913-925.

Shen, X., Nakamura, H., 2013. Local interfacial velocity measurement method using a four-sensor probe. Int. J. Heat Mass Transf. 67, 843-852. doi:10.1016/j.ijheatmasstransfer.2013.08.064 
Shen, X., Saito, Y., Mishima, K., Nakamura, H., 2005. Methodological improvement of an intrusive foursensor probe for the multi-dimensional two-phase flow measurement. Int. J. Multiph. Flow 31, $593-$ 617. doi:10.1016/j.ijmultiphaseflow.2005.02.003

Tian, D., Yan, C., Sun, L., Liu, G., 2014. Local interfacial parameter distribution for two-phase flow under rolling conditions using a four-sensor optical probe. Ann. Nucl. Energy 66, 124-132. doi:10.1016/j.anucene.2013.12.006

Tomiyama, A., Tamai, H., Zun, I., Hosokawa, S., 2002. Transverse migration of single bubbles in simple shear flows. Chem. Eng. Sci. 57, 1849-1858. doi:10.1016/S0009-2509(02)00085-4

Wilcox, D., 1998. Turbulence modeling for CFD, Journal of Chemical Information and Modeling. DCW industries, La Canada, CA.

Wu, Q., Ishii, M., 1999. Sensitivity study on double-sensor conductivity probe for the measurement of interfacial area concentration in bubbly flow. Int. J. Multiph. Flow 25, 155-173.

Wu, Q., Welter, K., McCreary, D., Reyes, J.N., 2001. Theoretical studies on the design criteria of doublesensor probe for the measurement of bubble velocity. Flow Meas. Instrum. 12, 43-51. doi:10.1016/S0955-5986(00)00041-8

Xue, J., 2004. BUBBLE VELOCITY, SIZE AND INTERFACIAL AREA MEASUREMENTS IN BUBBLE COLUMNS, PhD thesis.

Zhang, Z., 2010. LDA application methods: laser Doppler anemometry for fluid dynamics. Springer Science \& Business Media. 